\title{
Surgical Outcomes of Contrast Sensitivity and Visual Acuity in Uveitis-Associated Cataract
}

\author{
Atsunobu Takeda ${ }^{1,2}$ \\ Eiichi Hasegawa' \\ Shoji Notomi ${ }^{1,2}$ \\ Keijiro Ishikawa' \\ Mitsuru Arima ${ }^{1,2}$ \\ Yusuke Murakami \\ Shintaro Nakao' \\ Toshio Hisatomi ${ }^{3}$ \\ Koh-Hei Sonoda ${ }^{1,4}$ \\ 'Department of Ophthalmology, \\ Graduate School of Medical Sciences, \\ Kyushu University, Fukuoka, Japan; \\ ${ }^{2}$ Department of Ophthalmology, Clinical \\ Research Institute, National Hospital \\ Organization, Kyushu Medical Center \\ Fukuoka, Japan; ${ }^{3}$ Department of \\ Ophthalmology, Chikushi Hospital, \\ Fukuoka University, Fukuoka, Japan; \\ ${ }^{4}$ Department of Ocular Pathology and \\ Imaging Science, Graduate School of \\ Medical Sciences, Kyushu University, \\ Fukuoka, Japan
}

Purpose: To evaluate the pre- and post-operative outcomes of phacoemulsification in patients with uveitis-associated cataract in remission, such as conventional visual acuity (VA), photopic and mesopic contrast visual acuity (CVA), and flares in the anterior chamber objectively assessed as intraocular inflammation.

Patients and Methods: This prospective study included 26 eyes of 19 patients with uveitis and 45 eyes of 26 controls who underwent cataract surgery at the Kyushu University Hospital and Kyushu Medical Center in Fukuoka, Japan, from October 2016 to December 2018. Conventional VA and flare values in the anterior chamber were evaluated preoperatively and 1 and 3 months postoperatively. Photopic and mesopic CVAs were assessed preoperatively and 3 months postoperatively.

Results: The best-corrected VA (BCVA) was improved significantly from baseline to 1 and 3 months postoperatively in both groups ( $P<0.01$ in both groups). The mean preoperative $100 \%$ and $10 \%$ CVAs under the photopic condition were significantly lower in the uveitis group than in the control group $(P<0.05$ for both CVA), whereas the mean preoperative $100 \%$ CVA under the mesopic condition was comparable between the two groups. Although the mean preoperative $100 \%$ and $10 \%$ CVAs improved significantly from baseline under both photopic and mesopic conditions in both groups $(P<0.01$ in both groups), the postoperative contrast sensitivities under both photopic and mesopic conditions remained lower in the uveitis group than in the control group ( $P<0.01$ for both conditions). The postoperative complications included recurrence of active inflammation in five eyes and cystoid macular edema in one eye and were managed by topical steroid therapy alone.

Conclusion: Cataract surgery for uveitis-associated cataracts during remission is well tolerated. However, the present results suggest that amelioration of hemeralopia and/or nyctalopia is not as good as expected after cataract surgery in patients with uveitis.

Keywords: cataract, phacoemulsification, uveitis, contrast sensitivity, photopic and mesopic conditions

\section{Introduction}

Cataract is an opacification and cloudiness of the lens that blurs the light passing through the lens to the retina, which decreases not only visual acuity (VA) but also contrast sensitivity (CS), and thus results in a reducing quality of vision. Cataract develops due to chronic intraocular inflammation and adverse effects of long-term corticosteroid treatment in addition to aging in patients with uveitis. ${ }^{1}$ Cataract surgery can improve visual impairment in patients with uveitis for both agematched controls. However, such patients are at high risk of postoperative complications, such as macular edema, and consequent visual loss. ${ }^{2,3}$
Correspondence: Atsunobu Takeda Department of Ophthalmology, Graduate School of Medical Sciences, Kyushu University, 3-I-I Maidashi, Higashi-Ku,

Fukuoka, 8I2-8582, Japan

Tel +8I-92-642-5648

Fax +8I-92-642-5663

Email takeda.atsunobu.248@m.kyushu-u. ac.jp 
Visual acuities can be assessed via several different parameters such as best-corrected visual acuity (BCVA) and contrast sensitivities (CS). ${ }^{4} \mathrm{CS}$ have the ability to detect relative differences in luminance and distinguish details, edges, or borders of an object from its background. ${ }^{5}$ CS are affected in many eye disorders such as cataract, glaucoma, retinitis pigmentosa, diabetic retinopathy, macular diseases, and optic neuropathies. ${ }^{6,7}$ Cataract is the most common disease that impairs CS in the elderly, ${ }^{8}$ suggesting that cataract surgery facilitates management of $\mathrm{CS}$ in elderly uveitis patients. Furthermore, CS impairment under photopic and mesopic conditions is observed in patients with diabetes mellitus, even in those without symptoms of diabetic retinopathy, because of ganglion cell and inner retinal dysfunction. ${ }^{9} \mathrm{CS}$ is impaired in patients with Behçet's disease without ocular complications because of presumed cranial parenchymal or vascular involvement. ${ }^{10}$ Therefore, although many patients exhibit good VA in the routine vision test after cataract surgery, some, such as those with uveitis, may experience difficulty seeing due to retinal dysfunction, leading to CS impairment.

A long-term quiescence of intraocular inflammation before cataract surgery can reduce recurrence of intraocular inflammation in patients with uveitis. ${ }^{1,11}$ Several procedures have been reported to avoid exacerbation or relapse of intraocular inflammation in uveitis. $^{12}$ Preoperative prophylaxis by topical and/or systemic administration of corticosteroids predisposes uveitis patients to favorable results after cataract surgery. ${ }^{3}$ Despite management steps, complications of cataract surgery still occur in patients with uveitis. However, few prospective studies have assessed the improvement of visual function before and after cataract surgery using these prophylactic procedures and the use of a hydrophobic acrylic lens in patients with uveitis. ${ }^{13}$

In this study, we aimed to evaluate the pre- and postoperative outcomes of small-incision phacoemulsification with implantation of hydrophobic acrylic lenses such as conventional visual acuities (VAs) and contrast visual acuity (CVA) under photopic and mesopic conditions in patients with uveitis. In addition, aqueous flare values in the anterior chamber were objectively assessed as intraocular inflammation by using a laser flare cell meter in patients with uveitis.

\section{Patients and Methods}

This prospective study included 26 eyes of 19 patients with uveitis and 45 eyes of 26 controls at the
Department of Ophthalmology, Kyushu University Hospital and Kyushu Medical Center from October 2016 to December 2018.

This study was conducted in accordance with the guidelines of the Declaration of Helsinki. This was approved by the Institutional Review Board of Clinical Research at the Kyushu University and Kyushu Medical Center and registered on University Medical Information Network (UMIN ID: 000041141). We obtained written informed consent from all the participants before performing any study procedures or examinations. Randomization was not performed because the participants were divided into two groups: non-uveitis as the negative control and uveitis. Participants were not blinded because they underwent the same cataract surgery in both the groups.

In this study, the number of target cases was set at 90 eyes: 45 eyes with uveitis and 45 non-uveitis eyes. We set targets according to the outcomes of previous reports. ${ }^{2,12}$ When we assumed improvement of visual acuity ( $\log$ MAR) at $0.5 \pm 0.35$ in the uveitis group ${ }^{12}$ and 0.3 \pm 0.25 in the control group, ${ }^{2}$ we estimated the number of the necessary eyes according to significant difference between the uveitis and the control group in Student's $t$-test. However, it was difficult to enroll enough patients to reach the target numbers in the uveitis group because there were few patients with uveitis in whom cataract surgery alone was enough to improve their visual acuity.

\section{Inclusion Criteria}

Patients aged older than 50 years who were required to undergo cataract surgery with IOL implantation were included. Before surgery, it was a prerequisite for eligibility of patients with uveitis to have a quiescence of intraocular inflammation for more than 3 months in the absence of systemic immunosuppressive therapy. In cases with vitritis, cells may persist even in inactive stage and cannot be completely eliminated.

\section{Exclusion Criteria}

The exclusion criteria were as follows:

1. Medical history of any of the following conditions that were likely to affect surgical outcome:

Preoperative trauma, pre-existing macular pathologies, vitreous hemorrhage, diabetes mellitus, rhegmatogenous retinal detachment, ocular surface disorders, glaucoma uncontrolled by medical therapy, and optic atrophy. 
1. Corneal endothelial cell density less than 1000 cells $/ \mathrm{mm}^{2}$.

2. Long-term use of systemic corticosteroids and immunosuppressants.

3. Presence of systemic and ocular complications that make it difficult to perform cataract surgery.

4. History of ocular surgery in the eligible eye.

\section{Preoperative Examinations}

Objective evaluation of posterior synechia and lens nucleus hardness was performed using slit-lamp examination. Endothelial cell count (ECC) was assessed preoperatively (ECC-pre) and postoperatively at 3 months (ECC-post). Central corneal ECC was assessed using a noncontact Tomey EM-3000 specular microscope (Tomey Corp, Nagoya, Japan). The percentage of postoperative endothelial cell loss (ECLoss $\%)$ was calculated as previously described: ${ }^{14}$ ECLoss (\%) = ([ECC-pre - ECC-post] $/$ ECC-pre) $\times 100$, where ECLoss $\%$ is the percentage of postoperative endothelial cell loss, ECC-pre is the preoperative cell count, and ECC-post is the corneal ECC 3 months postoperatively.

\section{Surgical Technique}

Cataract surgeries were performed by one surgeon at each institutes. Local anesthesia was administered using topical $4 \%$ lidocaine and/or peribulbar $2 \%$ lidocaine anesthesia was delivered. Both $0.5 \%$ tropicamide and $0.5 \%$ phenylephrine hydrochloride were topically applied 90, 60, 45, 30, and 15 minutes before surgery to dilate the eye. For small incision cataract surgery, a side port entry was made at the 2 and 10 o'clock position with $22.5^{\circ}$ angle knife. Approximately $0.15 \mathrm{~mL}$ of Opegan- $\mathrm{Hi}^{\circledR}{ }^{\circledR}$ (sodium hyaluronate $1 \%$; Santen Pharmaceuticals Co. Ltd, Osaka, Japan) was injected into the anterior chamber in all patients. Patients who had posterior synechia underwent posterior synechialysis. The patients underwent a 2.75 clear corneal incision followed by continuous curvilinear capsulorhexis. All of the surgeries were performed using Ozil torsional hand piece with the Infiniti Vision System (Alcon). Phacoemulsification was performed with $100 \%$ torsional ultrasound, $200 \mathrm{mmHg}$ vacuum, and a $30 \mathrm{cc} / \mathrm{min}$ aspiration rate. Following phacoemulsification, 1 type of intraocular hydrophobic acrylic lens (PCB00V, Johnson \& Johnson Vision Care, Inc.) was inserted into the capsular bag in both groups.

\section{Postoperative Care Routine}

At the end of the surgery, all patients received subconjunctival injection of $1.65 \mathrm{mg}$ of dexamethasone and amikacin sulfate. From day one after the surgery, $0.1 \%$ disodium dexamethasone eye drops were used to suppress postoperative inflammation four times a day for one week and tapered to three times a day for three weeks. $0.1 \%$ bromfenac sodium hydrate eye drops were also given twice a day for three months. Routine antibiotic prophylaxis with $0.3 \%$ gatifloxacin eye drops 4 times a day for four weeks was also administered. Patients in both groups received the same treatment.

\section{Outcome Measures}

The primary outcome of this study was the best-corrected distance visual acuity (BCVA). BCVA was measured monocularly using Landolt ring chart (system charts SC2000 Nidek Instruments, Gamagori, Japan) at a distance of $5 \mathrm{~m}$ one day before operation and at one month and three months after operation. All tests were performed at a constant background luminance of $150 \mathrm{~cd} / \mathrm{m}^{2}$. The secondary outcomes of this study were eye inflammation (laser flare cell meter), contrast sensitivities (CS), and incidence of adverse events. CS were measured with $100 \%$ and 10\% CVA under both photopic and mesopic conditions with the contrast sensitivities acuity tester (CAT-CP) (NEITZ CO, Nagoya, Japan) as previously described. ${ }^{15}$ CVA was determined monocularly under four conditions; one day before operation and at three months after operation. Landolt rings are used with the CAT-CP, and the luminance of the rings and background can be chosen between photopic $\left(100 \mathrm{~cd} / \mathrm{m}^{2}\right)$ or mesopic $\left(5 \mathrm{~cd} / \mathrm{m}^{2}\right)$. The luminance of $100 \mathrm{~cd} / \mathrm{m}^{2}$ and $5 \mathrm{~cd} / \mathrm{m}^{2}$ was the physiologic conditions for daytime and nighttime, respectively. Measurements were made after darkadaption for at least 5 minutes, and the refractive error was corrected for $5 \mathrm{~m}$. The decimal visual acuity (VA) values were converted to $\operatorname{logMAR}$ units, and mean values were used for the analyses. In patients with decimal VA was $>1.3 \log$ MAR units on CAT-CP, CVA was defined as $1.4 \log$ MAR units because CAT-CP can measure $\leq 1.3$ $\log$ MAR units.

Laser flare-cell meter (LFCM) measurements were performed with the FM-600 laser flare cell meter (Kowa, Tokyo, Japan) to evaluate aqueous flare in the anterior chamber objectively on one day before operation, at one month and three months after operation as previously described. ${ }^{16}$ Measurements were taken with the pupil dilated (using phenylephrine $2.5 \%$ and cyclopentolate $0.1 \%$ ) in a dark room. The mean value of 5 valid laser flare measures with a background scatter of less than $15 \%$ 
was calculated and was used as the baseline flare value for analysis. Laser flare values were shown in photon per millisecond (ph/ms).

The final outcome was measured according to the amount of change when comparing preoperative and postoperative VA, CVA, and the mean aqueous flare values.

Objective evaluation included slit-lamp examination to assess the cell intensity in the aqueous humor according to the SUN working Group criteria, ${ }^{17}$ between one week and three months after cataract surgery. Relapse of active inflammation was diagnosed when the following clinical evidence was observed: (1) An increase by at least two stages of cellularity and/or flare (opacity) in the anterior segment of the eye, at slit-lamp examination, compared with the baseline level (necessarily 0-1), and/or (2) An increase by at least two stages of vitreal inflammation, at slit-lamp examination, compared with the baseline level (necessarily $0-1$ ).

\section{Statistical Analysis}

The data were analyzed using Jump software version 15 (Business Unit of SAS, Cary, NC). Fisher's exact test was used to evaluate differences in categorical variables, like the $\mathrm{M} / \mathrm{F}$ ratio, between the two groups. Both of the preand postoperative BCVA and CVA were converted to $\log$ MAR for statistical analysis. Student's $t$-test or a paired $t$-test was used to compare normally and equally distributed data. Mann-Whitney's $U$-test or a Wilcoxon signed-rank test was performed to compare data that were not distributed normally or equally. Chi-square test was used to compare lens nucleus hardness. The tests were also used to detect the difference between the conditions of patients in both groups preoperatively, and postoperatively at one and/or three months. A $P$ value of $<0.05$ was considered to be significant.

\section{Results}

The demographic data of both groups are shown in Table 1 . A total of 26 eyes of 19 patients with uveitis (6 men and 13 women; mean age, $64.5 \pm 9.30$ years) and 45 eyes of 26 patients with non-uveitis as control (11 men and 15 women; mean age, $74.4 \pm 6.84$ years) fulfilled the inclusion criteria and were enrolled in this prospective study (Table 1). Since cataract arises earlier in patients with uveitis than those with age-matched controls, the mean age of the uveitis group was significantly younger than that of the control group ( $P=0.0002$, Student's $t$-test) (Table 1$)$. The study group comprised a heterogeneous group of uveitic entities,
Table I Patients' Characteristics

\begin{tabular}{|l|l|l|l|}
\hline & Uveitis & Control & P value \\
\hline Patients, n & 19 (26 eyes) & 26 (45 eyes) & \\
\hline $\begin{array}{l}\text { Age at surgery, years } \\
\text { Mean } \pm \text { SD } \\
\text { Range }\end{array}$ & $\begin{array}{l}64.5 \pm 9.30 \\
50-82\end{array}$ & $\begin{array}{l}74.4 \pm 6.84 \\
57-85\end{array}$ & $0.0002^{\S}$ \\
\hline Male gender & $6(32 \%)$ & $\mathrm{II}(42 \%)$ & $0.32^{\dagger}$ \\
\hline $\begin{array}{l}\text { Uveitis diagnosis (M/F), n } \\
\text { Sarcoidosis }\end{array}$ & $1 / 6$ & N/A & \\
$\begin{array}{l}\text { Behcet's disease } \\
\text { Vogt-Koyanagi-Harada } \\
\text { disease } \\
\text { Uveitis due to } \\
\text { nontuberculous } \\
\text { mycobacterium } \\
\text { Varicella-zoster virus- } \\
\text { associated iridocyclitis } \\
\text { Unknown }\end{array}$ & $0 / 2$ & N/A & \\
\hline
\end{tabular}

Notes: ${ }^{\S}$ Student's $t$-test. ${ }^{\dagger}$ Fisher's exact test.

including 15 patients with panuveitis and 4 with anterior uveitis. The largest single diagnosis group consisted of panuveitis; it included by sarcoidosis $(n=7)$, Behçet's disease $(\mathrm{n}=4)$, Vogt-Koyanagi-Harada $(\mathrm{VKH})$ disease ( $\mathrm{n}$ $=2$ ), and uveitis due to nontuberculous mycobacterium (NTM) $(n=2)$. The diagnosis of anterior uveitis comprised patients with varicella-zoster virus-associated iridocyclitis $(\mathrm{n}=1)$ and uveitis with unknown etiology $(\mathrm{n}=3)$. The comparison of data of the anterior pole of the eyes including the numbers of eyes with preoperative lens opacity grade $\geq$ III (Emery-Little Classification System) and posterior synechia, pre- and postoperative central corneal ECCs, percentage of corneal endothelial cell loss, and surgical time is shown in Table 2. There was no significant between-group difference in lens nucleus hardness, pre- and postoperative central corneal ECCs, percentage of corneal endothelial cell loss, or surgical time. Posterior synechia was observed in three eyes of three patients with uveitis for which the etiologies were VKH, sarcoidosis, and unknown granulomatous uveitis.

We did not allocate one eye in the uveitis group because we were unable to insert IOL due to the rupture of the Zinn's zonule. Furthermore, we excluded four eyes of three patients from our analysis of visual outcome in the uveitis group because of pre-existing macular degeneration. In total, 66 eyes completed the baseline and postoperative examinations in this study. 
Table 2 Summary of the Eyes' Demographic Data of the Anterior Pole for Visual Acuity Analyses

\begin{tabular}{|c|c|c|c|}
\hline & $\begin{array}{l}\text { Uveitis } \\
(n=21)\end{array}$ & $\begin{array}{l}\text { Control } \\
(n=45)\end{array}$ & $P$ value \\
\hline $\begin{array}{l}\text { Preoperative lens } \\
\text { opacity grade } \\
\text { (Emery-Little } \\
\text { Classification } \\
\text { System) } \geq \text { III, n }\end{array}$ & 7 (33.3\%) & II (24.4\%) & $0.86 *$ \\
\hline Posterior synechia, $\mathrm{n}$ & 3 (II.5\%) & $0(0 \%)$ & \\
\hline ECC-pre $\left(/ \mathrm{mm}^{2}\right)$ & $2588.1 \pm 294.5$ & $2671.2 \pm 238.6$ & $0.15^{\S}$ \\
\hline ECC-pro $\left(/ \mathrm{mm}^{2}\right)$ & $2506.6 \pm 355.0$ & $2487.9 \pm 304.4$ & $0.73^{\S}$ \\
\hline ECLoss (\%) & $2.22 \pm 8.52$ & $6.60 \pm 8.19$ & $0.14^{\S}$ \\
\hline $\begin{array}{l}\text { Total surgical time } \\
\text { (min.) }\end{array}$ & $17.14 \pm 9.85$ & $17.11 \pm 6.30$ & $0.98^{\S}$ \\
\hline
\end{tabular}

Notes: *Chi-square test. 'Student's $t$-test.

Abbreviations: ECC-pre, preoperative central corneal endothelial cell count; ECC-pro, postoperative central corneal endothelial cell count; ECLoss, percentage of corneal endothelial cell loss.

\section{Visual Outcomes}

The mean preoperative $\log$ MAR was significantly worse in the uveitis group than that in the control group (Figure 1, $P=0.0002$, Student's $t$-test). Both groups had statistically significant improvements on $\log$ MAR at one month and three months postoperatively over preoperative values (Figure 1, $P<0.0001$ for both groups, paired $t$-test). Postoperative $\log$ MAR was comparable at one and three months between the two groups (Figure 1, $P=0.071, P=$ 0.15 , Student's $t$-test). There was no statistical difference

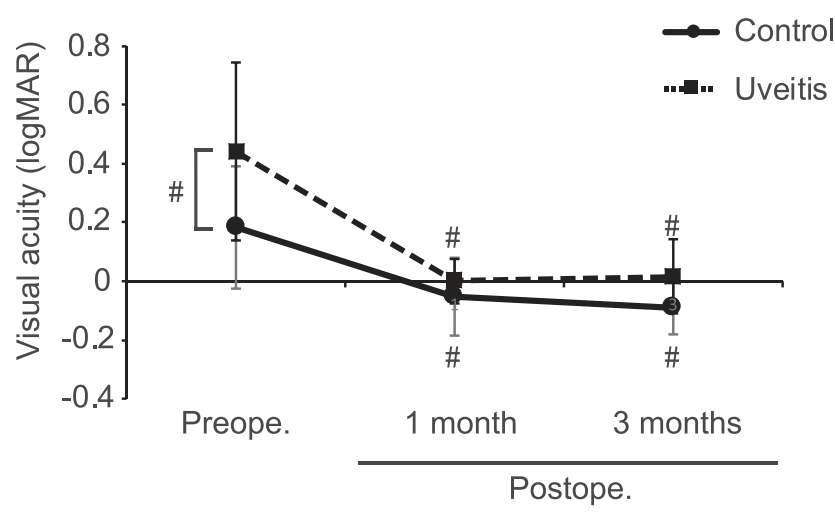

Figure I Graphs comparing the mean pre- and postoperative logMAR between uveitis and control groups. The pre- and postoperative best corrected visual acuity for distance converted to $\log M A R$. The mean preoperative $\log M A R[0.44 \pm 0.30$, $95 \%$ confidence interval $(\mathrm{Cl}): 0.33-0.55]$ was significantly decreased in the uveitis group than in the control group $(0.18 \pm 0.21,95 \% \mathrm{Cl}$ : $0.1 \mathrm{I}-0.26)$. The mean preoperative logMAR was significantly improved postoperatively at $\mathrm{I}$ and 3 months in both groups (I month, control: $-0.052 \pm 0.13,95 \% \mathrm{Cl}$ : $-0.097--0.0079$; uveitis: $0.025 \pm 0.075,95 \% \mathrm{Cl}:-0.037-0.087 ; 3$ months, control: $-0.089 \pm 0.090,95 \% \mathrm{Cl}$ : $-0.15-0.029$, uveitis: $0.0091 \pm 0.13,95 \% \mathrm{Cl}:-0.073-0.091)$. Error bars represent the standard error (SE). ${ }^{\#} P<0.01$; Student's $t$-test or paired $t$-test. in any visual outcome parameter, including IOP, between the control and uveitis groups at three months follow-up (data not shown).

\section{Contrast Sensitivities}

The mean preoperative $100 \%$ and $10 \%$ CVA were significantly lower under photopic conditions in the uveitis group than those in the control group $(P=0.0007$ and $P=$ 0.0002 , respectively, Student's $t$-test) (Figure 2A). However, there was no significant difference in the mean preoperative $100 \%$ CVA under mesopic conditions between the two groups ( $P=0.057$, Student's $t$-test) (Figure 2B), although the mean preoperative 10\% CVA under mesopic conditions was significantly low in the uveitis group compared to the control group $(P=0.021$, Student's $t$-test) (Figure 2B).

At three months postoperatively, the mean postoperative $100 \%$ and $10 \%$ CVA were significantly lower for both photopic and mesopic conditions in the uveitis group than those in the control group (Figure 2A and $\mathrm{B}, P<0.0001$ and $P<$ 0.0001 for photopic conditions, $P=0.0016$ and $P=0.0010$ for mesopic conditions, Student's $t$-test), although the mean $100 \%$ and $10 \%$ CVA under both photopic and mesopic condition significantly improved from baseline in both the groups (Figure 2A and $\mathrm{B}, P<0.0001$ for both conditions in the control group, $P<0.0001$ and $P=0.0002$ for photopic conditions in the uveitis group, $P<0.0001$ and $P<0.0001$ for mesopic conditions in the uveitis group, paired $t$-test). We then compared the degree of improvement in $100 \%$ and $10 \%$ CVAs under both photopic and mesopic conditions between the two groups. There was no significant between-group difference in the degree of improvement in $100 \%$ and $10 \%$ CVAs under either the photopic or mesopic condition (Figure 2C; $P=0.96$ and $P=0.49$ for photopic conditions and $P=0.46$ and $P=0.98$ for mesopic conditions, respectively, Student's $t$-test).

\section{Flare Analysis}

As shown in Figure 3, there was a statistically significant difference in preoperative flare values measured with LFCM between the uveitis and control groups $(P=0.021$, MannWhitney's $U$-test). At one and three months postoperatively, the flare values were significantly higher in the uveitis group than those in the control group $(P=0.011$ and $P=0.017$, respectively, Mann-Whitney's $U$-test).

Although there was not statistically significant difference in the uveitis group, the mean flare values were significantly increased compared to the baseline at one 

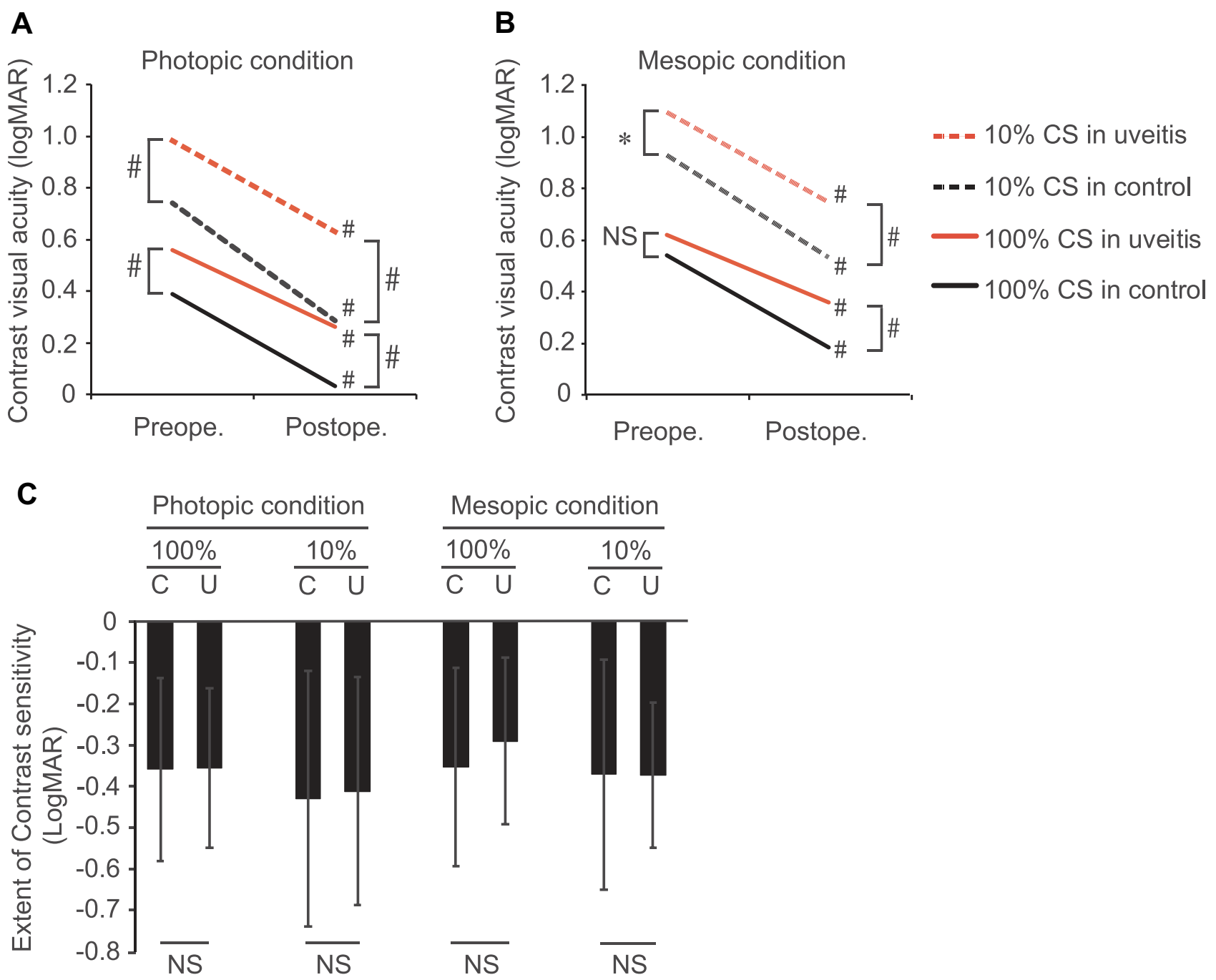

Figure 2 Comparison of the mean pre- and postoperative (A) photopic and (B) mesopic contrast visual acuity (CVA) and (C) the mean degree of improvement in CVA under photopic and mesopic conditions after cataract surgery between the uveitis and control groups. The pre- and postoperative contrast visual acuity for distance converted to logMAR. The mean preoperative $100 \%$ and $10 \%$ contrast visual acuities were decreased under photopic condition in the uveitis group ( $100 \%$ CVA: $0.55 \pm 0.29$, 95\% Cl: 0.4I-0.68; 10\% CVA: $0.98 \pm 0.31,95 \%$ Cl: 0.83-I.12) compared to those in the control group (100\% CVA: $0.39 \pm 0.22,95 \%$ Cl: $0.31-0.47 ; 10 \%$ CVA: $0.74 \pm 0.27$, $95 \% \mathrm{Cl}: 0.65-0.83$ ). The mean preoperative 10\% CVA under mesopic conditions was significantly lower in the uveitis group than in the control group (control: $0.93 \pm 0.26$, 95\% Cl: 0.84-I.0I; uveitis: I.06 $\pm 0.25,95 \% \mathrm{Cl}: 0.93-\mathrm{I} .18$ ), whereas the mean preoperative $100 \%$ CVA was not (uveitis: $0.57 \pm 0.3 \mathrm{I}, 95 \% \mathrm{Cl}$ : $0.43-0.72$; control: $0.54 \pm 0.25$, $95 \% \mathrm{Cl}: 0.45-0.62)$. At 3 months postoperatively, the mean postoperative $100 \%$ and $10 \%$ CVAs were decreased under both photopic and mesopic conditions in the uveitis group (100\% and 10\% CVAs under photopic condition: $0.23 \pm 0.19,95 \% \mathrm{Cl}: 0.14-0.33$ and $0.61 \pm 0.27,95 \%$ Cl: $0.48-0.74$, respectively; $100 \%$ and I0\% CVAs under mesopic condition: $0.35 \pm 0.22,95 \% \mathrm{Cl}: 0.24-0.47$ and $0.74 \pm 0.21,95 \% \mathrm{Cl}: 0.62-0.85$, respectively) compared to those in the control group (I00\% and $10 \% \mathrm{CVAs}$ under photopic condition: $0.034 \pm 0.15,95 \% \mathrm{Cl}:-0.012-0.086$ and $0.29 \pm 0.18,95 \% \mathrm{Cl}: 0.22-0.35$, respectively; $100 \%$ and I0\% CVAs under mesopic condition: $0.18 \pm 0.18,95 \% \mathrm{Cl}$ : $0.12-$ 0.24 and $0.53 \pm 0.17,95 \% \mathrm{Cl}: 0.47-0.59$, respectively). (C) The mean degree of improvement in CVA was comparable between the uveitis (100\% and $10 \%$ CVAs under photopic condition: under both photopic and mesopic conditions $-0.36 \pm 0.19,95 \% \mathrm{Cl}:-0.46-0.25$ and $-0.4 \mathrm{I} \pm 0.28,95 \% \mathrm{Cl}$ : $-0.67--0.33$, respectively; I00\% and I0\% CVAs under mesopic condition: $-0.29 \pm 0.20,95 \% \mathrm{Cl}:-0.42--0.19$ and $-0.37 \pm 0.18,95 \% \mathrm{Cl}:-0.5 \mathrm{I}--0.24$, respectively) and control (I00\% and I0\% CVAs under photopic condition: $-0.36 \pm 0.22,95 \% \mathrm{Cl}:-0.43-0.29$ and $-0.43 \pm 0.3 \mathrm{I}, 95 \% \mathrm{Cl}:-0.54-0.32$, respectively; $100 \%$ and I0\% CVAs under mesopic condition: $-0.35 \pm 0.24$, $95 \% \mathrm{Cl}$ : $-0.42--0.28$ and $-0.37 \pm 0.28,95 \% \mathrm{Cl}:-0.45--0.29$, respectively) groups. Error bars represent the standard deviation (SD). $* P<0.05$, ${ }^{*} P<0.0 \mathrm{I}$; Student's $t$-test or paired t-test.

Abbreviation: NS, not significant.

month after surgery in the control and uveitis groups (Figure 3, $P<0.0001$ and $P=0.055$, Wilcoxon signedrank test). The flare values were still upregulated at three months after surgery compared to the baseline in both groups (Figure 3, $P<0.0001$ and $P=0.0039$, Wilcoxon signed-rank test).

\section{Postoperative Complications}

During the enrollment period, five eyes showed relapse of active inflammation after cataract surgery in patients with uveitis. One eye had cystoid macular edema (CME). All of the relapse of uveitis and CME developed between two and three months after cataract surgery. Relapse of active 
Preope.

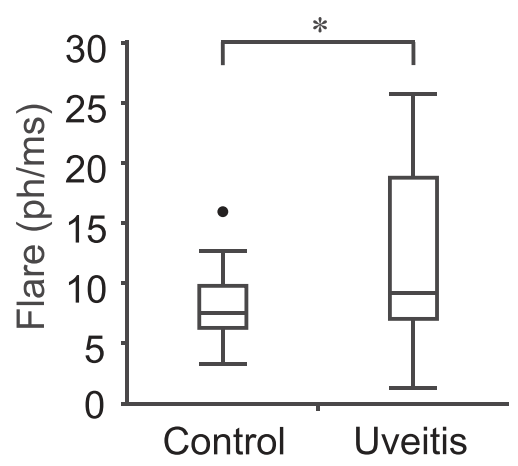

1 Mo postope.

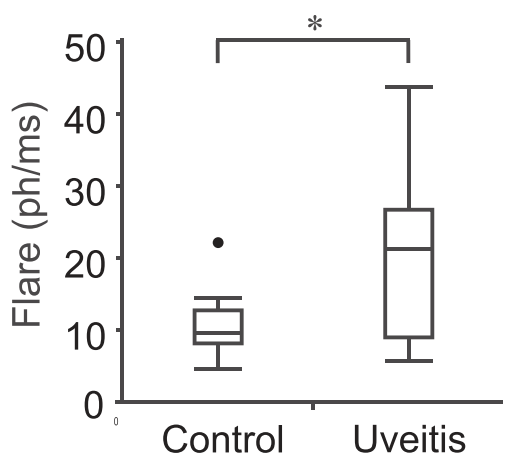

3 Mo postope.

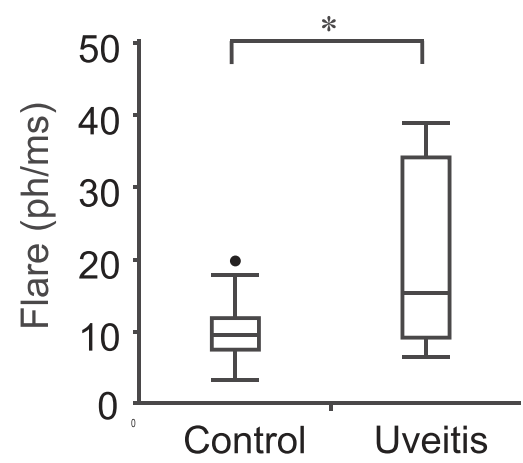

Figure 3 Comparison of aqueous flare values between the control and uveitis groups over time. The mean preoperative flare values [0.44 \pm 0.30 , interquartile range (IQR): 0.33-0.55] was significantly increased in the uveitis group (uveitis: 9.I, IQR: 7.0-18.8) compared to that in the control group (control: 7.5, IQR: 6.2-9.8). At I and 3 months postoperatively, the mean flare values were significantly increased in the uveitis group (I month: 2I.3, IQR: 9.0-26.8; 3 months: I5.4, IQR: 9.I-34.I) compared to those in the control group (I month: 9.6, IQR: 8.2-12.8; 3 months: 9.6, IQR: 7.6-II.9). The mean flare values at 3 months postoperatively were still higher than those at baseline in both groups. Laser flare values are shown in photon per millisecond $(\mathrm{ph} / \mathrm{ms})$. $* P<0.05$; Mann-Whitney $U$-test.

Abbreviation: Mo, month.

inflammation was addressed by increasing the frequency of eye drops of $0.1 \%$ betamethasone sodium phosphate. The CME was addressed by sub-Tenon injection of triamcinolone acetonide. The underlying cause of recurrent uveitis and CME was sarcoidosis in 3 eyes, 1 eye with NTM, and 1 eye with unknown etiology for the recurrence, and VKH in CME. No other complications such as posterior synechia, hypotony, posterior capsule opacity (PCO) development, and IOP elevation were observed.

\section{Discussion}

In this study, we found that the degree of improvement in CS after cataract surgery in patients with uveitis was inferior to that in patients in the control group, although the improvement in BCVA was comparable between the two study groups. The decline of CS is reported in patients with cataract who have comparable conventional visual acuities compared to those without cataract. ${ }^{18}$ Cataract surgery can improve CS under both photopic and mesopic conditions. ${ }^{19}$ In our study, most of the patients with uveitis had panuveitis with retinal lesions, including sarcoidosis, and VKH as well as Behçet's disease. Retinal periphlebitis in posterior uveitis of sarcoidosis is sometimes subclinically observed and only seen on fluorescein angiography, ${ }^{20}$ indicating that retinal dysfunction may start to arise at an early stage of sarcoidosis uveitis. In addition, VKH patients in this study had 'sunset glow fundus' appearance, which is reported to be associated with worse retinal sensitivity due to photoreceptor impairment. $^{21}$ Therefore, these results suggest that the changes in CS under both photopic and mesopic conditions can be the early symptoms of retinal disorders or visual dysfunction in panuveitis due to dysfunction of retinal cells by uveitis. Furthermore, amelioration of hemeralopia and/or nyctalopia is not as good as expected after cataract surgery in patients with uveitis.

Although the quiescence of intraocular inflammation had been achieved for at least three months before cataract surgery, postoperative aqueous flare was increased in patients with uveitis compared to controls. In addition, the recurrence of uveitis and $\mathrm{CME}$ emerged after cataract surgery. ${ }^{22,23}$ Immunosuppression for perioperative prophylaxis to avoid relapse or recurrence has been validated. Systemic corticosteroid therapy can suppress perioperative inflammation compared to topical corticosteroid therapy, ${ }^{24}$ suggesting that the systemic administration of immunosuppressants could have prevented postoperative complications such as recurrence of intraocular inflammation and CME in uveitis in this present study. However, another prospective long-term study that compared the efficacy between systemic and intensive topical corticosteroid therapy revealed that the effects of intensive topical corticosteroid therapy are comparable to those of systemic corticosteroid therapy. ${ }^{25}$ A single sub-Tenon's triamcinolone acetonide injection is as effective in control of uveitis relapse as postoperative 4-week oral corticosteroid

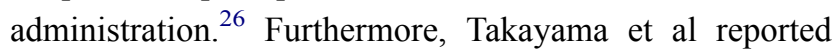
that regional steroid therapy alone can control relapse of uveitis in older uveitis patients. ${ }^{2}$ Systemic corticosteroids induce various systemic adverse effects such as hyperglycemia, hypertension, and osteoporosis. ${ }^{27}$ Minimally invasive procedures performed during cataract surgery such as 
small-incision phacoemulsification are effective in obtaining satisfactory results with less frequent postoperative complications; ${ }^{2}$ this suggests that prophylactic systemic corticosteroid therapy is not necessary before and after the minimally invasive cataract surgery, at least in most of older uveitis patients in whom the quiescence of intraocular inflammation had been achieved for at least 3 months before cataract surgery.

A previous comparison of various materials of IOL implantation revealed that postoperative complications are less frequent in uveitis patients with implantation of acrylic lenses than those with silicone lenses. ${ }^{13,28}$ In our study, although postoperative laser flare values were increased despite the hydrophobic acrylic lens implantation, there were no postoperative complications such as posterior synechiae, hypotony, and elevated IOP. Therefore, these results suggest that the implantation of hydrophobic acrylic lens contributes to the prevention of complications in patients with uveitis, resulting in better visual outcomes. Postoperative complications such as posterior capsule opacity were not observed because the median time of onset of PCO was over one year after cataract surgery. ${ }^{29}$ Thus, long-term follow-up will be necessary to detect postoperative complications such as PCO.

There are some limitations to this study. First, the sample size was small. Multicenter studies with a larger sample size may be required to clarify whether decreased contrast sensitivities are associated with uveitis patients. Second, this study included patients older than 50 years who required cataract surgery. The severity of postoperative inflammation in uveitis patients might be milder in younger uveitis patients. ${ }^{11}$ In addition, impairment of CS was gradually reduced by aging. ${ }^{30}$ Therefore, the inclusion criteria might have caused selection bias.

In conclusion, cataract surgery for uveitis patients can obtain favorable outcomes with less postoperative complications after a long-term quiescence of intraocular inflammation. However, there are cases where CVA may not improve as much as expected levels under both photopic and mesopic condition after cataract surgery in uveitis patients.

\section{Data Sharing Statement}

The datasets used and/or analyzed during the current study are available from the corresponding author on reasonable request.

\section{Acknowledgment}

We would like to thank to Dr. Naoya Hashiguchi, Ms. Tomomi Soda, Ms. Asami Iwashita, Ms. Momoko Mizokami, and Ms. Keiko Kojima for their excellent technical support throughout clinical examinations for visual acuities.

\section{Author Contributions}

All authors contributed to data analysis, drafting or revising the article, have agreed on the journal to which the article will be submitted, gave final approval of the version to be published, and agreed to be accountable for all aspects of the work.

\section{Funding}

This study was supported by a grant from Japan Society for the Promotion of Science (JSPS) KAKENHI Grant Number 21K09745 [grants C (A.T.)] from the Ministry of Education, Science, Sports and Culture, Japan, and grants from Novartis Pharma Research Grants (AT; Tokyo, Japan) Alcon Pharma Research Grants (AT; Tokyo, Japan). The funding sources had no role in the design and conduct of the study; collection, management, analysis, and interpretation of the data; preparation, review, or approval of the manuscript; or the decision to submit the manuscript for publication.

\section{Disclosure}

We have read the journal's policy and the authors of this manuscript have the following competing interests: This study was conducted in collaboration with Kyushu University, Kyushu Medical Center, and AMO Japan, Ltd. Takeda A and Sonoda K-H received research support fees from AMO Japan, Ltd. Murakami Y reports grants from Grants-in-Aid for Scientific Research from KAKENHI (\#JP19K09952), The Center for Clinical and Translational Research of Kyushu University Hospital, The Cell Science Research Foundation; personal fees from Novartis Pharmaceutical Co. Ltd, Bayer Yakuhin Ltd, Santen Pharmaceutical Co. Ltd, AMO Japan Co. Ltd, Senju Pharmaceutical Co. Ltd, and Otsuka Pharmaceutical Co. Ltd, outside the submitted work. The authors report no other conflicts of interest in this work.

\section{References}

1. Llop SM, Papaliodis GN. Cataract surgery complications in uveitis patients: a review article. Semin Ophthalmol. 2018;33(1):64-69. doi:10.1080/08820538.2017.1353815 
2. Takayama K, Fujii S, Ishikawa S, Takeuchi M. Short-term outcomes of coaxial microincision cataract surgery for uveitis-associated cataract without postoperative systemic steroid therapy. Ophthalmologica. 2014;231(2):111-116. doi:10.1159/000355491

3. Elgohary MA, McCluskey PJ, Towler HM, et al. Outcome of phacoemulsification in patients with uveitis. Br J Ophthalmol. 2007;91 (7):916-921. doi:10.1136/bjo.2006.109660

4. Qin VL, Conti FF, Singh RP. Measuring outcomes in cataract surgery. Curr Opin Ophthalmol. 2018;29(1):100-104. doi:10.1097/ICU.000 0000000000434

5. Chung ST, Legge GE. Comparing the shape of contrast sensitivity functions for normal and low vision. Invest Ophthalmol Vis Sci. 2016;57(1):198-207. doi:10.1167/iovs.15-18084

6. Trick GL, Burde RM, Cordon MO, Santiago JV, Kilo C. The relationship between hue discrimination and contrast sensitivity deficits in patients with diabetes mellitus. Ophthalmology. 1988;95(5):693-698. doi:10.1016/S0161-6420(88)33125-8

7. Marmor MF. Contrast sensitivity versus visual acuity in retinal disease. Br J Ophthalmol. 1986;70(7):553-559. doi:10.1136/bjo.70. 7.553

8. Nomura H, Ando F, Niino N, Shimokata H, Miyake Y. Age-related change in contrast sensitivity among Japanese adults. Jpn J Ophthalmol. 2003;47(3):299-303. doi:10.1016/S0021-5155(03)00 011-X

9. Safi S, Rahimi A, Raeesi A, et al. Contrast sensitivity to spatial gratings in moderate and dim light conditions in patients with diabetes in the absence of diabetic retinopathy. BMJ Open Diabetes Res Care. 2017;5(1):e000408. doi:10.1136/bmjdrc-2017-000408

10. Ozsoy E, Cankaya C, Ozturk E, Gunduz A, Ulucan PB. Measurement of contrast sensitivity in patients with Behcet's disease without ocular involvement. Korean J Ophthalmol. 2019;33(2):167-172. doi:10.33 41/kjo.2018.0099

11. Baheti U, Siddique SS, Foster CS. Cataract surgery in patients with history of uveitis. Saudi $J$ Ophthalmol. 2012;26(1):55-60 doi:10.1016/j.sjopt.2011.10.003

12. Agrawal R, Murthy S, Ganesh SK, Phaik CS, Sangwan V, Biswas J. Cataract surgery in uveitis. Int J Inflam. 2012;2012:548453. doi:10.11 $55 / 2012 / 548453$

13. Alio JL, Chipont E, BenEzra D, Fakhry MA; International Ocular Inflammation Society SGoUCS. Comparative performance of intraocular lenses in eyes with cataract and uveitis. J Cataract Refract Surg. 2002;28(12):2096-2108. doi:10.1016/S0886-3350(02)01452-9

14. Mahdy MA, Eid MZ, Mohammed MA, Hafez A, Bhatia J. Relationship between endothelial cell loss and microcoaxial phacoemulsification parameters in noncomplicated cataract surgery. Clin Ophthalmol. 2012;6:503-510. doi:10.2147/OPTH.S29865

15. Oomachi K, Ogata K, Sugawara T, Hagiwara A, Hata A, Yamamoto S. Evaluation of contrast visual acuity in patients with retinitis pigmentosa. Clin Ophthalmol. 2011;5:1459-1463. doi:10. 2147/OPTH.S23070

16. Krall EM, Arlt EM, Jell G, et al. Intraindividual aqueous flare comparison after implantation of hydrophobic intraocular lenses with or without a heparin-coated surface. J Cataract Refract Surg. 2014;40(8):1363-1370. doi:10.1016/j.jcrs.2013.11.043

17. Jabs DA, Nussenblatt RB, Rosenbaum JT; Standardization of Uveitis Nomenclature Working G. Standardization of uveitis nomenclature for reporting clinical data. Results of the First International Workshop. Am J Ophthalmol. 2005;140(3):509-516.

18. Elliott DB. Evaluating visual function in cataract. Optom Vis Sci. 1993;70(11):896-902. doi:10.1097/00006324-199311000-00006

19. Trueb PR, Albach C, Montes-Mico R, Ferrer-Blasco T. Visual acuity and contrast sensitivity in eyes implanted with aspheric and spherical intraocular lenses. Ophthalmology. 2009;116(5):890-895. doi:10. 1016/j.ophtha.2008.12.002

20. Rothova A. Ocular involvement in sarcoidosis. $\mathrm{Br} J$ Ophthalmol. 2000;84(1):110-116. doi:10.1136/bjo.84.1.110

21. Abu El-Asrar AM, Al Mudhaiyan T, Al Najashi AA, et al. Chronic recurrent Vogt-Koyanagi-Harada disease and development of 'Sunset Glow Fundus' predict worse retinal sensitivity. Ocul Immunol Inflamm. 2017;25(4):475-485. doi:10.3109/09273948.2016.1139730

22. Singh K, Shrestha S, Manandhar A. Outcome of cataract surgery in eyes with uveitis. Nepal J Ophthalmol. 2019;11(22):152-157. doi:10.3126/nepjoph.v11i2.27820

23. Takai N, Kobayashi T, Kida T, Ikeda T. Clinical features of Japanese patients with ocular inflammation and their surgical procedures over the course of 20 years. Clin Ophthalmol. 2020;14:2799-2806. doi:10.2147/OPTH.S273938

24. Meacock WR, Spalton DJ, Bender L, et al. Steroid prophylaxis in eyes with uveitis undergoing phacoemulsification. Br J Ophthalmol. 2004;88(9):1122-1124. doi:10.1136/bjo.2003.032482

25. Mora P, Gonzales S, Ghirardini S, et al. Perioperative prophylaxis to prevent recurrence following cataract surgery in uveitic patients: a two-centre, prospective, randomized trial. Acta Ophthalmol. 2016;94 (6):e390-394. doi:10.1111/aos.12955

26. Rosel E, Tsakos G, Bernabe E, Sheiham A, Bravo M. Assessing the level of agreement between the self- and interview-administered Child-OIDP. Community Dent Oral Epidemiol. 2010;38(4):340-347. doi:10.1111/j.1600-0528.2010.00533.x

27. Jabs DA, Rosenbaum JT, Foster CS, et al. Guidelines for the use of immunosuppressive drugs in patients with ocular inflammatory disorders: recommendations of an expert panel. Am J Ophthalmol. 2000;130(4):492-513. doi:10.1016/S0002-9394(00)00659-0

28. Mehta S, Linton MM, Kempen JH. Outcomes of cataract surgery in patients with uveitis: a systematic review and meta-analysis. Am J Ophthalmol. 2014;158(4):676-692 e677. doi:10.1016/j.ajo.2014. 06.018

29. Abbouda A, Tortorella P, Restivo L, Santoro E, De Marco F, La Cava M. Follow-up study of over three years of patients with uveitis after cataract phacoemulsification: outcomes and complications. Semin Ophthalmol. 2016;31(6):532-541. doi:10.3109/08820538. 2015.1009554

30. Owsley C, Sekuler R, Siemsen D. Contrast sensitivity throughout adulthood. Vision Res. 1983;23(7):689-699. doi:10.1016/0042-69 89(83)90210-9
Clinical Ophthalmology

\section{Publish your work in this journal}

Clinical Ophthalmology is an international, peer-reviewed journal covering all subspecialties within ophthalmology. Key topics include: Optometry; Visual science; Pharmacology and drug therapy in eye diseases; Basic Sciences; Primary and Secondary eye care; Patient Safety and Quality of Care Improvements. This journal is indexed on PubMed
Central and CAS, and is the official journal of The Society of Clinical Ophthalmology (SCO). The manuscript management system is completely online and includes a very quick and fair peer-review system, which is all easy to use. Visit http://www.dovepress.com/ testimonials.php to read real quotes from published authors. 